\title{
Two-step etch method to improve the filtering performance of nuclear track membrane
}

\author{
WANG Yang ${ }^{1, a}$ QU Hua ${ }^{1, b}$ MO Dan ${ }^{2, c}$ \\ ${ }^{1}$ Tianjin Polytechnic University, Tianjin 300387, China \\ ${ }^{2}$ Institute of Modern Physics, Chinese Academy of Sciences, Lanzhou 730000, China \\ awangy0419@163.com, ${ }^{\text {b } q u h u a-930 @ 163 . c o m, ~}{ }^{\mathrm{c} m o d a n @ i m p c a s . a c . c n}$
}

Keywords: Nuclear track membrane, Two-step etch method, Double-cone shape, Flux comparison, Filtering performance

\begin{abstract}
A method for the fabrication of double-cone shape pores in polyethylene terephthalate (PET)-based nuclear track membranes (NTMs) is presented here. PET films of $25 \mu \mathrm{m}$ thickness were irradiated with $\mathrm{Bi}$ ions (kinetic energy $9.5 \mathrm{MeV} / \mathrm{u}$, fluence $5 \times 107$ ions $/ \mathrm{cm} 2$ ) at room temperature under vacuum at the Heavy Ion Research Facility (HIRFL) in Lanzhou.Then using two-step etch method to fabricate the NTMs with double-cone pores. Comparing the filtering flux by using Yellow-river water between the double-cone shape and regular, cylindrical shape in the experiment. Filtering flux through simulation by ANSYS Fluent and the water test results are also compared. The experimental results show that the flux of double-cone shape is significantly 5 times higher than that of cylindrical shape. And NTMs with inner pore size of $450 \mathrm{~nm}$ can effectively separate bacteria by an average of $99.90 \%$ (particle counter) in raw water.
\end{abstract}

\section{Introduction}

At present, there are many ways in water treatment. Nanofiltration (NF) and reverse osmosis primarily target the separation of ions, ultrafiltration (UF) targets the separation of molecules, and microfiltration (MF) targets the separation of particles and microorganisms[1-5]. And membrane systems are receiving increased attention for water treatment and other applications[6].

Nuclear track membranes (NTMs), a kind of promising new filtration material due to the advantages of ascendant feature and ideal structure, are produced from thin polymer films irradiated by heavy ion beam from a cyclotron/accelerators techniques[7]. Latent tracks of heavy ions in polymer material are treated by chemical etching and as a result, membranes with regular, cylindrical pores with diameter in the range of $0.02-3.0 \mu \mathrm{m}$ and number of pores up to 1010 pores per $\mathrm{cm} 2$ are commercially available in various dimensions including rolls of different lengths and widths up to $50 \mathrm{~cm}$ [8]. NTMs have attracted significant attention owing to their utility in laboratory and industrial filtration, biological sciences, medicine, pharmaceutical production, purification technology, food industry and other highly specific applications.

In recent years, many studies have been published about NTMs filtration with some significant results. S. Guo[9] developed the basic nature of nuclear track membranes for liquid media in 1998. Charles R Martin's group turned to the study of NTMs with conical pores[10-12]. Z. Lisheng et al.[13] have expounded the connection between the pore size and the particle size. Also, many computational fluid dynamics (CFD) simulations have been used to evaluate the microfiltration process[14-16].

All in all, most studies were based on the NTMs with a regular, cylindrical pore shape, but the filtering performance through improving the pore shapes is rarely involved. We have developed the fabrication of different pore shapes before[17], which includes double-cone shape, funnel shape, pencil shape, spherical funnel and cup shape. Nonetheless, research on their performance is still lacking. In the present paper, based on our prior research, we carried out experimental research of using two-step etch method to fabricate the NTMs with double-cone pores, compared their filtering performance and simulated the filtering performance by ANSYS Fluent. 
Now in China, with the rapidly development of agriculture and industry, the problems of water pollution are getting worse and make people keep an eye on them. Particularly in some remote mountainous areas and rural areas, the problems of drinking-water are more and more serious. So our researches have an important significance in the application of NTMs and water treatment field for the future.

\section{Organization of the Text}

\subsection{Experimental}

\subsubsection{Sample fabrication}

PET films of $25 \mu \mathrm{m}$ thickness were irradiated with Bi ions (kinetic energy $9.5 \mathrm{MeV} / \mathrm{u}$, fluence $5 \times 10^{7}$ ions $/ \mathrm{cm}^{2}$ ) at room temperature under vacuum at the Heavy Ion Research Facility (HIRFL) in Lanzhou. Irradiated PET films were etched with methanol-free or methanol-containing alkaline solutions at different etching steps. Reference [17] has gave very readable introductory discussions. Two different etching conditions (Table 1) were used only for etching PET samples. The etching unit, made up of a JK-MP-5H super thermostatic water tank and a magnetic stirrer, ensures the accurate, steady etching temperature with an error in $\pm 0.5^{\circ} \mathrm{C}$.

Table 1 Etching conditions.

\begin{tabular}{lllll}
\hline $\begin{array}{l}\text { Etching } \\
\text { condition }\end{array}$ & Etchant solution & $\begin{array}{l}\text { Temperature } \\
\left({ }^{\circ} \mathrm{C}\right)\end{array}$ & $\begin{array}{l}\text { Bulk etch rate } \\
(\mathrm{nm} / \mathrm{min})\end{array}$ & $\begin{array}{l}\text { Track etch rate } \\
(\mathrm{nm} / \mathrm{min})\end{array}$ \\
\hline Condition 1 & $1 \mathrm{M} \mathrm{NaOH}$ & 80 & 15 & 800 \\
Condition 2 & $\begin{array}{l}9 \mathrm{M} \mathrm{NaOH} \\
(\text { methanol/water 1:1) }\end{array}$ & 20 & 42 & 400 \\
\hline
\end{tabular}

To obtain a regular, cylindrical pore shape, irradiated PET films were placed under a UV light illumination[18] for 1 hour, then etched in condition 1 for $31 \mathrm{~min}$. These samples were numbered Sample A.

To obtain a double-cone pore shape, PET films were etched by two-step etch method: etching two cones from both sides to a certain depth then annealing the track. First, irradiated PET films were etched in condition 2 for 20 min. Second, PET films after a preliminary etching were etched in condition 1 for 26 min. These samples were numbered Sample B.

\subsubsection{Filtration}

The experimental facility and methods of filtering the suspending particulate substances and bacteria in water by using NTMs agreed approximately with previous results[9][19]. The filter facility adopts an oil-free vacuum pump and a $1000 \mathrm{~mL}$ quartz filtration unit, which is made up of a cylindrical funnel, a quartz filter and a clamp.

After filtering the Yellow-river water sample carefully, record the time employed in filtering equal volume of water sample. The experiment was repeated three times then the average fluid velocity was calculated.

\subsection{ANSYS Simulation}

ANSYS Fluent 14.5 software has been applied to simulate and analyze the filtration experiment. Simulation models of NTMs are in Fig.1. There are 81 pores in the unit area, with a distance of 1 $\mu \mathrm{m}$ between adjacent pores. And the length of the holes is $25 \mu \mathrm{m}$.
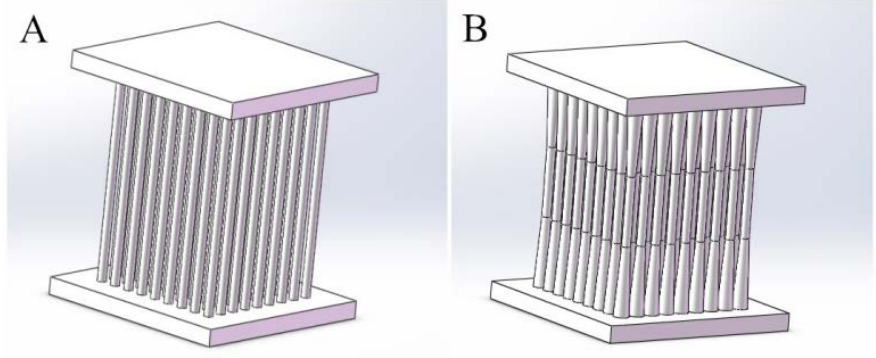

Fig.1 Simulation models of NTMs.

(A) Cylindrical shape model, (B) Double-cone shape model

Setting parameters that inner-pore diameters are $450 \mathrm{~nm}$ and the filtering liquid is water with 
bacteria. Under the same experimental conditions, including pressure, temperature, boundary and relaxation factors. The contours of total pressure and the contours of velocity magnitude are shown in Fig.2 and Fig.3.

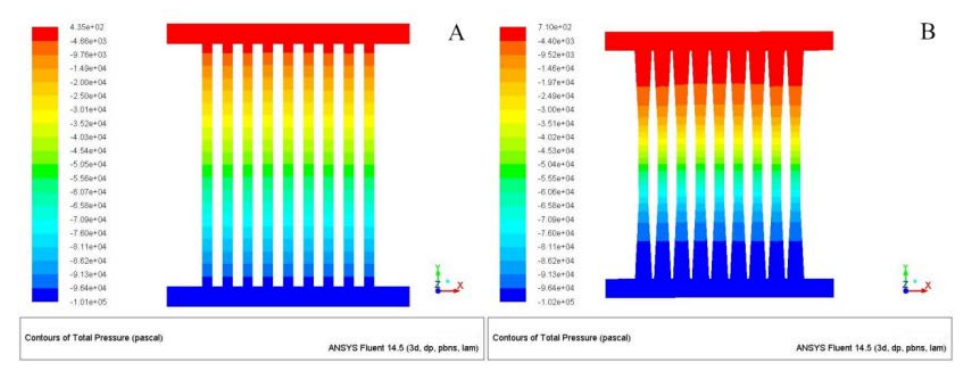

Fig.2 Contours of total pressure (Pa).

(A) Cylindrical shape, (B) Double-cone shape

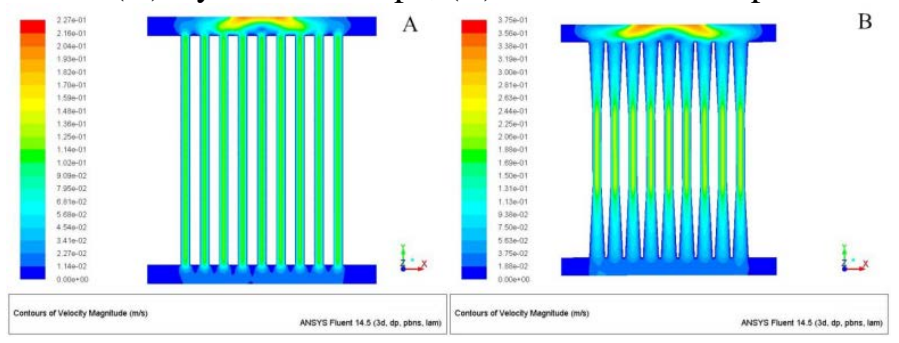

Fig.3 Contours of velocity magnitude $(\mathrm{m} / \mathrm{s})$.

\subsection{Results and discussion}

(A) Cylindrical shape, (B) Double-cone shape

\subsubsection{Sample Analysis}

Two kinds of pore shapes under SEM are shown in Fig. 4 and Fig. 5, from which we can find the inner-pore sizes are both $450 \mathrm{~nm}$. When fabricating NTMs samples, the temperature and concentration of the etchant have an important influence on the pore size, especially there is a significant exponential relationship between the temperature and pore size[20]. All in all, it is essential to control different etching conditions in order to fabricate unique pore shapes.

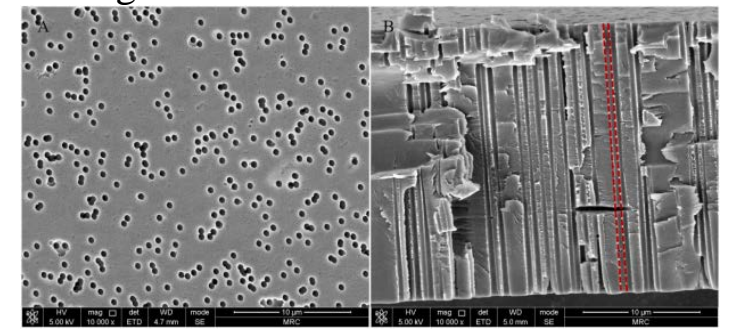

Fig.4 Cylindrical pores under SEM.

(A) Surface micrograph, (B) Section micrograph

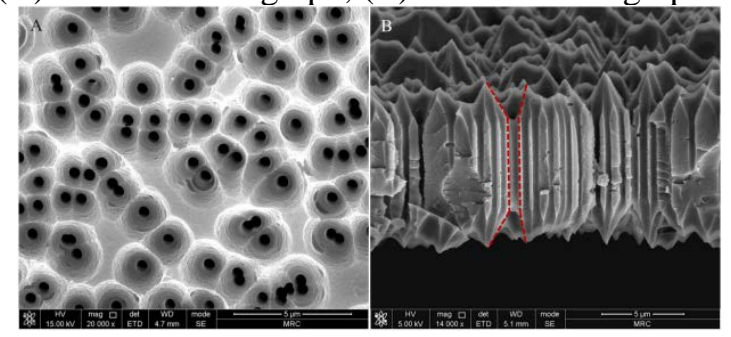

Fig.5 Double-cone shape under SEM.

(A) Surface micrograph, (B) Section micrograph

When fabricating Sample A, the purpose of UV light illumination is to greatly improve the track etch rate $\boldsymbol{v}_{\boldsymbol{t}}$ and to remove the cone angles. When fabricating Sample $\boldsymbol{B}$, two-step etch method was used not only to fabricate the double-cone shape pores, but to strictly control the inner-pores diameter. In the process of fabrication, as the film becomes thinner after preliminary etching, the conduction arises, which leads to a shorter etching time compared with Sample A.

Bubble pressure method[21-22], by which one can measure the inner-pore size distribution accurately though 3H-2000PB pore size analyzer (Beishide Instrument Technology Co., Ltd.) , was 
used to measure samples. The results were shown in Fig.6, from which one can see the pores of two kinds of samples exhibit a uniform distribution and the inner-pores diameters are of the same size in principle.

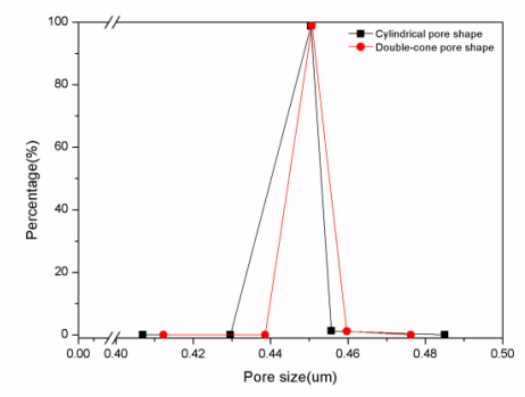

Fig.6 Pore-size distribution.

\subsubsection{Filtering Performance Analysis}

After meticulous measurements with distilled and filtered water, under circumstances of extreme environmental cleanliness, we have demonstrated that Poiseuille's formula:

$$
V=\frac{\pi k^{4}}{128 \eta L}\left(P_{1}-P_{2}\right) n t
$$

In the expression, $V$ is the volume of liquid having viscosity $\eta$ passing through a NTM of pore size $k$, pore number $n$, pore length $L$ under a pressure difference $\left(P_{1}-P_{2}\right)$ for a period of time $t$.

The relation between fluid velocity and volume in Fig.7 was obtained by this experiment.

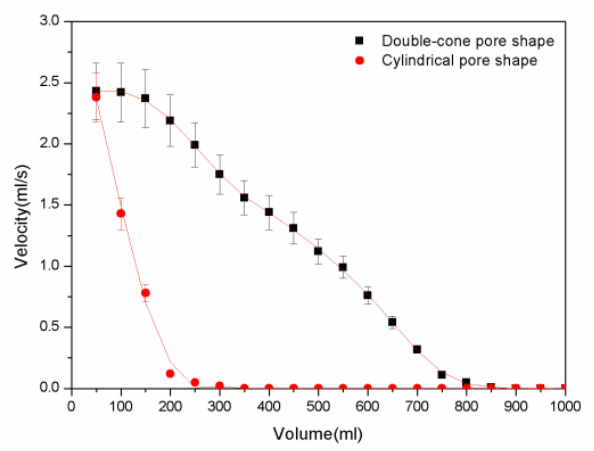

Fig.7 The relation between fluid velocity and volume.

Fig.7 shows the relation between fluid velocity and volume of two kinds of NTMs. It seems that the initial filtration velocity would be the same since there is no pore blockage at the start of the experiment, which appears to be consistent with that physical picture. Soon afterwards, the differences of the flux is obvious. As for cylindrical shape, with the holes being blocked fleetly and entirely, the flux is almost zero when the water volume gets $250 \mathrm{ml}$. The inner-hole blocking is the main cause of membrane fouling meanwhile. As for double-cone shape, the water flux and water volume are significantly higher. And the holes are not entirely blocked until $850 \mathrm{ml}$. Four main reasons for this obvious advantage are following:

(1) The overlapping holes in the surface increase the porosity of the membrane. The liquid can be gathered into holes faster under the same pressure gradient.

(2) Water can't be trapped and impurities are not easy to block at the entrance. According to T. Ando et al.[23], at first, the impurity particles were adsorbed on the entrance of cone holes, instead of the entrance of inner-holes. Next, according to B.Xiong et al.[24] and C. He et al.[25], the cake layer clogging is suggested to be the primary membrane fouling. But for Sample B, the cake layer is not so compact to block the holes due to the rough surface (Fig.5B).

(3) Flux of Sample B rises with increasing pore size when water running-off.

(4) According to the Poiseuille's formula (Formula. 1), the flux rises because of the reducing of total pore length and inner-pore length of Sample $\boldsymbol{B}$.

After filtering, the colonies number, chromaticity and turbidity of water samples were tested. 
The test results are located in Table 2.

Table 2 Results of water sample.

\begin{tabular}{|c|c|c|c|}
\hline Samples & Chromaticity/ & Turbidity / JTU & Colonies number / CFU $\cdot \mathrm{mL}^{-}$ \\
\hline Raw water & $378.5 \pm 9.8$ & $79.09 \pm 5.72$ & $4.8 \times 10^{4}$ \\
\hline After precipitate & $21.4 \pm 6.5$ & $8.199 \pm 1.341$ & $3.5 \times 10^{4}$ \\
\hline $\begin{array}{l}\text { After filtering by } \\
\text { cylindrical pores } \\
\text { sample }\end{array}$ & $1.212 \pm 0.278$ & $0.303 \pm 0.175$ & Less than 100 \\
\hline $\begin{array}{l}\text { After filtering by } \\
\text { double-cone pores } \\
\text { sample }\end{array}$ & $0.911 \pm 0.195$ & $0.303 \pm 0.234$ & Less than 100 \\
\hline $\begin{array}{l}\text { Standards for } \\
\text { drinking water } \\
\text { quality } \\
\text { (GB 5749-2006) }\end{array}$ & 15 & 1 & 100 \\
\hline
\end{tabular}

As can be seen from Table 2, Yellow River water pollution was very serious with high chromaticity, turbidity and large colonies number. After precipitation treatment for raw water sample, the chromaticity and turbidity of water deposited[26] is rather lower, but the colonies number is still considerable. After filtering by Sample A and Sample B, both the colonies number, chromaticity and turbidity reduced. Thus, Sample $\boldsymbol{B}$ can get to the bacteria filtering standard (refer to Standards for drinking water quality GB 5749-2006) by using two-step etch method.

\subsubsection{Simulation Analysis}

The results of analysis show that the fluid in cylindrical holes have roughly the same velocity. But in the double-cone holes, more fluid have gathered at the entrance. Then the flow rate increases dramatically at the inner holes. Combined with Table 3, it can be seen clearly that the mass flux of double-cone holes significantly larger than that of cylindrical holes.

The results showed that the simulation data coincident well with the experimental results.

Table 3 Mass flow rate.

\begin{tabular}{ll}
\hline Pore shapes & Flux $(\mathrm{kg} / \mathrm{s})$ \\
\hline Cylindrical pores & $1.0267571 \times 10^{-9}$ \\
Double-cone pores & $4.8858215 \times 10^{-9}$ \\
\hline
\end{tabular}

\subsection{Conclusion}

In the experiment, while under the etching condition that the lower temperature and higher concentration, double-cone shape samples can be also fabricated by single etch method, it can be very difficult to control the inner-pore size according to etch time. Nevertheless, two-step etch method can be invoked as the preferred method to fabricate NTMs with unusual pore shape in respect that the inner-pore size is strictly controlled. The filtering performance of NTMs by two methods was observed and compared. The evident advantage of double-cone shape is the high flux, high accuracy, the anti-blocking performance as well as high efficiency and filtering standard. During sample fabrication, it is necessary to control the etching temperature and etching time strictly according to the $v_{t}$ and $v_{g}$, in order to control the inner-pore size.

As a new type of filtration material, NTM is non-pollution, washable and recycled. Therefore, improving the filtering performance of NTM without sacrificing filtering quality is of wide application and scientific implication in the water treatment field, which can encourage the development of its application. 


\section{Acknowledgments}

This work was supported by the National Natural Science Foundation of China (No.11205215), Institute of Modern Physics, Chinese Academy of Sciences and Wuwei Kejin Xinfa Technology Co.,Ltd..

\section{References}

[1] B. Xiong et al. Fouling behavior of dissolved organic matter in nanofiltration membranes from a pilot-scale drinking water treatment plant: An autopsy study, Water Res. 99(2016) 162-170.

[2] C. Xu et al. Preparation of PES ultrafiltration membranes with natural aminoacids based zwitterionic antifouling surfaces, Appl. Surf. Sci. 385 (2016) 130-138.

[3] C. He, R.D.Vidic. Application of microfiltration for the treatment of Marcellus Shale flowback water: Influence of floc breakage on membrane fouling, J. Membr. Sci. 510 (2016) 348-354.

[4] K. J. Howe, M.M. Clark, Fouling of microfiltration and ultrafiltration membrane by natural waters, Environ. Sci. Technol. 36 (2002) 3571-3576.

[5] K. Akamatsu, W. Lu, T. Sugawara, S. Nakao, Development of a novel fouling suppression system in membrane bioreactor using an intermittent electric field, Water Res. 44 (2010) 825-830.

[6] H. Ma et al. Membrane fouling reduction by backpulsing and surface modification, J. Membr. Sci. 173 (2000) 191-200.

[7] M. Buczkowski et al. Radiation resistance of track etched membranes, Radiat. Meas. 34 (2001) 597-599.

[8] S.N. Husaini et al. Application of nuclear track membrane for the reduction of pollutants in the industrial effluent, Radiat. Meas. 43 (2008) S607-S611.

[9] Guo S L. Basic nature of nuclear track membranes for liquid media. New. Astron. Rev. 42 (1998) 205-216.

[10] P. Scopece et al. Conical nanopore membranes: solvent shaping of nanopores, NANOTECHNOLOGY. 17 (2006) 3951-3956.

[11] Li N C et al. Conical Nanopore Membranes. Preparation and Transport Properties, Anal. Chem. 76 (2004) 2025-2030.

[12] H. Mukaibo et al. Controlling the Length of Conical Pores Etched in Ion-Tracked Poly(ethylene terephthalate) Membranes, SMALL, 5 (2009) 2474-2479.

[13] Z. Lisheng et al. Study on filtration properties of nuclear track membrane and its blocking effect, Radiat. Meas. 43 (2008) S632-S634.

[14] K.L. Tung, C.J. Chuang. Effect of pore morphology on fluid flow and particle deposition on a track-etched polycarbonate membrane, Desalination 146 (2002) 129-134.

[15] M.M. Kim, A.L. Zydney. Effect of electrostatic, hydrodynamic, and Brownian forces on particle trajectories and sieving in normal flow filtration, J. Colloids Interface Sci. 269 (2004) 425431.

[16] M. Rahimi, S.S. Madaeni, M. Abolhasani, A.A. Alsairafi. CFD and experimental studies of fouling of a microfiltration membrane, Chem. Eng. Process: Process Intens. 48 (2009) 1405-1413.

[17] D. Mo et al. Fabrication of different pore shapes by multi-step etching technique in ion-irradiated PET membranes, Nucl. Instrum. Meth. B. 333 (2014) 58-63.

[18] Z. Zhu et al. Role of UV light illumination and DMF soaking in production of PET ion track membranes, Nucl. Instrum. Meth. B. 217 (2004) 449-456. 
[19] Guo S L, Hao X H, Lin J G, et al. Manipulation and effectiveness of bacteria filtration with particle track microfilters, Nuclear Tracks and Radiat. Meas. 19 (1991) 809-812.

[20] Samoilova L I, Apel P Y. Etching of small pores in PETP by different alkalis, Radiat. Meas. 25 (1995) 717-720.

[21] D. Hopkinsonetal. The bubble point of supported ionic liquid membranes using flat sheet supports, J. Membr. Sci. 468 (2014) 155-162.

[22] A. Shariati et al. Bubble point pressures of binary system of methanol and methyl propionate, Fluid Phase Equilibria 417 (2016) 166-170.

[23] T. Ando et al. Simulation of fouling and backwash dynamics in dead-end microfiltration: Effect of pore size, J. Membr. Sci. 392-393 (2012) 48-57.

[24] B. Xiong et al. Fouling of microfiltration membranes by flowback and produced waters from the Marcellus shale gas play, Water Res. 99 (2016) 162-170.

[25] C. Heetal. Microfiltration in recycling of Marcellus Shale flowback water: Solids removal and potential fouling of polymeric microfiltration membranes, J. Membr. Sci. 462 (2014) 88-95.

[26] Z. Lai et al. Deposit analysis of water-wall tubes in a municipal solid waste grate incinerator, Appl. Therm. Eng. 66 (2014) 415-422. 